\title{
A Hybrid SLM Technique for PAPR Reduction in OFDM
}

\author{
Salini I V \\ Department of Electronics and \\ Communication Engineering \\ MES College of Engineering \\ Kuttippuram, Kerala, India
}

\author{
Rino P C \\ Department of Electronics and \\ Communication Engineering \\ MES College of Engineering \\ Kuttippuram, Kerala, India
}

\begin{abstract}
With the advancement in age and its growing demands, there has been rapid growth in the field of communications. Multicarrier modulation is an attractive technique for fourth generation wireless communication. Orthogonal Frequency Division Multiplexing (OFDM) is one of the efficient multicarrier transmission schemes. OFDM consist of large number of independent sub carriers, as a result of which the amplitude of such a signal can have high peak values. The main drawback of OFDM system is that it suffers from high Peak to Average Power Ratio (PAPR). Due to high PAPR there is inefficient use of high power amplifier and this could limit transmission efficiency. Different technique can be used to reduce this PAPR; from that SLM is one of the efficient techniques. But the computational complexity and the transmission of side information are the concern problem in SLM. There for this paper, proposes a new SLM technique to reduce these drawbacks. As compared to the Conventional SLM method, the proposed Hybrid method provides less computational complexity and reduces the PAPR without any side information.
\end{abstract}

\section{Keywords}

BER (Bit Error Rate), CCDF (Complementary Cumulative Distribution Function), HPA (High Power Amplifier), OFDM (Orthogonal Frequency Division Multiplexing), PAPR (Peakto-Average Power Ratio), SLM (Selected Mapping), SI (Side Information).

\section{INTRODUCTION}

Communication is one of the important aspects of daily life. The ever increasing demand of this generation need for highspeed wireless transmissions have become more urgent than ever. In order to meet these demands, nowadays a various multi carrier modulation techniques have been developed, one of the noticeable technique among them being Orthogonal Frequency Division Multiplexing. OFDM is used in almost all current and forthcoming wireless communication standards (WiMAX, LTE Advanced) and digital broadcasting standards (DVB-T) [1]; it is also used in IEEE 802.11, IEEE 802.16, IEEE 802.20 standards [2].

OFDM is a frequency division multiplexing (FDM) scheme utilized as a digital multi carrier modulation method [3]. In which, large number of closely spaced orthogonal subcarriers are used to carry data. The data is divided into several parallel data streams or channels, one for each subcarrier. Each subcarrier is modulated with a conventional modulation scheme such as Quadrature Amplitude Modulation (QAM) or Phase Shift Keying (PSK) at a low symbol rate. The total data rate is to be maintained similar to that of the conventional single carrier modulation scheme with the same bandwidth.
One of the major drawback of OFDM is that its signal has high Peak-to-Average Power Ratio (PAPR) compared to a single carrier signal because an OFDM signal is the sum of many narrowband signals in the time domain [1][2]. However, an OFDM signal can have a high peak-to-average power ratio (PAPR) at the transmitter, which causes signal distortion such as in-band distortion and out-of band radiation due to the nonlinearity of the high power amplifier (HPA) and a worse bit error rate (BER) [4]. To avoid the performance degradation of OFDM signal due to high PAPR, the power amplifier (PA) must be operated in linear region with large dynamic range, where the power conversion is inefficient. This may leads to an adverse effect on battery life time in portable mobile devices, where the benefits of OFDM may be diminished by the drawback of high PAPR. A number of techniques have been proposed to deal with high PAPR problem. These techniques can be broadly classified into four categories [5]. Clipping [6], Coding [7], Constellation extension [1] [4] Multiple signal representation such as SLM [8] [9] and Partial Transmit Sequence (PTS) [10]. All of these methods have the ability to reduce the PAPR at the cost of system performances like distortion, bandwidth expansion, BER degradation, power increased etc. Therefore, the necessity to reduce the PAPR of normal OFDM signals by considering all of these factors and developing a system with good performance has been a challenge.

From the existing PAPR reduction method, Selected mapping (SLM) is one of the efficient techniques which reduce the peaks of a signal considerably. The drawback associated with this technique is its computational complexity and number of the bits for side information transmission.

The concentration of this paper is especially upon the SLM technique and its two drawbacks. To reduce these drawbacks of SLM, this paper proposes a hybrid technique by combining the ideas that was described in [11] and [12].

The rest of this paper is organized as follows: section 2 provides the basic OFDM system model, concept of PAPR and the significance of PAPR reduction. Section 3 gives the idea of conventional SLM technique. Section 4 proposes the new approach, section 5 gives the simulation results and finally section 6 concludes the paper.

\section{PAPR IN OFDM SYSTEM}

One of the key bullets in OFDM system is the existence of efficient and simple implementation of transmitter and receiver. Before explaining the PAPR, a simplified OFDM system model has been considered.

So the input complex-valued data of $\mathrm{N}$ subcarriers are described as, $X_{N}=\left\{X_{k}, k=0,1, \ldots . N-1\right\}$ is formed with each symbol modulating one of a set of subcarriers, $\left\{f_{k}, k=\right.$ 
$0,1 \ldots . N-1\}$ where $\mathrm{N}$ is the number of subcarriers and these are chosen to be orthogonal, that is, $f_{k}=\Delta f k$, where $\Delta f=1 / N T$ and $\mathrm{T}$ is the original symbol period[2]. Therefore, the transmitted OFDM signals can be written as,

$$
x(t)=\frac{1}{N T} \sum_{k=0}^{N-1} X_{k} e^{j 2 \pi f_{k t}}
$$

In OFDM system, a large number of independently modulated sub carriers are available. Due to the presence of that, the peak value of the signal power can be very high as compared to the average of the whole system. This ratio between the peak and the average power value is termed as Peak to Average Power Ratio [1] [2]. An OFDM signal consists of a number of separately modulated sub-carriers which can produces a large PAPR when added up coherently. When $\mathrm{N}$ signals with the same phase are added together, they produce a peak power that is $\mathrm{N}$ times the average power of the signal. For an OFDM signal with $\mathrm{N}$ sub carriers, the PAPR can be defined as,

$$
P A P R=\frac{\max _{0 \leq t \leq N T}|x(t)|^{2}}{\frac{1}{N T} \int_{0}^{N T}|x(t)|^{2}}
$$

For a baseband OFDM signal with $\mathrm{N}$ sub channel has,

$$
P A P R_{\max }=10 \log _{10} N
$$

$\mathrm{CDF}$ is one of the most frequently used performance measures for PAPR reduction techniques. CCDF is used to estimate the performance of any PAPR reduction techniques and it also used to calculate the bounds or the least number of redundancy bits that are essential to identify the PAPR sequences.

$$
\mathrm{CCDF}=\mathrm{P}(\mathrm{PAPR}>\mathrm{PAPRo})=1-(1-\exp (\mathrm{PAPRo}))
$$

It can also to reduce the total degradation by determine a proper output back-off of HPA. Moreover, it can directly apply distribution of PAPR to calculate the BER and estimate achievable information rates [2].

\subsection{Significance of PAPR Reduction in OFDM}

Today the mobile communication have an important role in our daily life, but the increasing energy cost of network operation is very high and that can make up as much as 50\% of the total operational cost. So the main source to minimise the energy cost is to increase the efficiency of HPA, at the radio frequency front end of the base station. But the efficiency of the HPA is directly related to the PAPR of the input signal [13]. For the transmission purposes most of the radio systems needs sufficient transmission power, so to get that much amount of transmission power they employs a HPA at the transmitter. When the HPA is operated at or near the saturation region, only if that time the system get high output power efficiency. Figure shows the nonlinear characteristics curve for power amplifier. But the nonlinear characteristic of the HPA is very sensitive to the changes in signal amplitudes. However, the variation of OFDM signal amplitudes is very wide with high PAPR. Therefore, the high PAPR of OFDM signals causes intermodulation between the subcarriers in HPA and introduces additional interference in to the system. This additional interference leads to an increase in BER. In order to reduce the signal distortion and keep a moderate $\mathrm{BER}$, it requires a linear work in its linear amplifier region with a large dynamic range. But most of the linear amplifier has poor efficiency and it is so expensive. So power efficient operation of the non-linear HPA with low back off values is an important issue. Hence, a better solution is to avoid the formation of such interference by reducing the PAPR of the transmitted signal.

Large PAPR also demands the DAC with enough dynamic range to accommodate the large peaks of the OFDM signals. Although, a high precision DAC supports high PAPR with a reasonable amount of quantization noise, but it is very expensive for a given sampling rate of the system. Whereas, a low-precision DAC would be cheaper, but its quantization noise will be significant, and as a result it reduces the signal Signal-to-Noise Ratio (SNR) when the dynamic range of DAC is increased to support high PAPR [2] [14].

When a HPA have a high dynamic range, it exhibits poor power efficiency. It has been shown that PAPR reduction can significantly save the power, in which the net power saving is directly proportional to the desired average output power and it is highly dependent upon the clipping probability level. Therefore, low efficiency is a strong motivation to reduce the PAPR in OFDM systems.

\section{CONVENTIONAL SLM TECHNIQUE}

SLM technique described first in [8] i.e., to be known as the classical SLM technique. In SLM technique the input data sequences are multiplied by each of the phase sequences and generate alternative new input symbol sequences. Each of these phase multiplied input data sequences is made the IFFT operation. Among them the one with the lowest PAPR is selected for transmission. Fig 1 shows the block diagram of the SLM technique at the transmitter end; here consider a single OFDM sequence $\mathrm{X}$ having a length of N. Each of this incoming OFDM sequence $\mathrm{X}$ is multiplied by $\mathrm{U}$ different rotation factors. Therefore $\mathrm{U}$ number of SLM sequence has been generated. These rotation factors are given as, $B^{(u)}=$ $b_{0}^{u}, b_{1}^{u}, \ldots \ldots \ldots b_{N-1}^{u}$ with $b_{n}^{u}=\left(e^{-j \theta_{n}^{i u}}\right), \quad$ where $\theta_{n}^{U}=[0,2 \pi), \theta$ is the angle of rotation, $\mathrm{n}=0,1,2 \ldots \ldots \mathrm{N}-1$ and $\mathrm{u}=1,2 \ldots \mathrm{U}$.

After multiplying these phase factors by the original OFDM sequence $X$, we get,

$$
X_{n}^{(u)}=X_{n} B_{n}^{(u)}
$$

The selection of multiplication factors has an important role. The rotation vectors (phase sequence) are used as side information so they are transmitted along with the signal to correctly recover the input signal at the receiver. This transmission of SI and the computational complexity are the drawback of this method. Different modifications have been takes place in this method to improve its performance [1] [2]. 


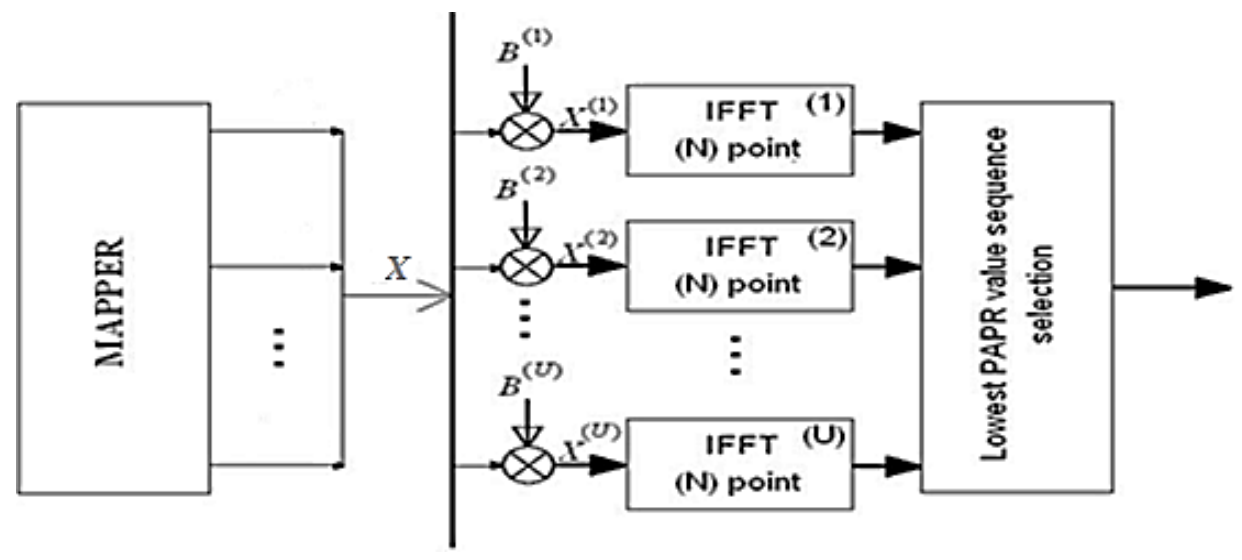

Fig 1: Conventional SLM technique at the transmitter end

\section{PROPOSED METHOD}

Computational complexity is one of the main drawbacks in conventional SLM. The computational complexity in SLM method is related to the IFFT blocks used in this method. If the number of sub-carriers increases the computational complexity also increased. For explanation, consider the OFDM system with $\mathrm{N}$ sub carriers. Computational complexity of the system in terms of complex addition can be described as [15],

$$
\text { Comlexity }=U N \log _{2} N
$$

In terms of multiplication complexity can be described as,

$$
\text { Comlexity }=U \frac{N}{2} \log _{2} N
$$

Where, $U$ is the number of newly generated sequence after the IFFT operation or it is the number phase sequences used to multiply the input signals. Hence, to reduce the complexity, reduce the number of computation of IFFT blocks. But the number of IFFT block is directly related to the number of subcarriers. So the reduction in number of subcarriers reduces the computation of IFFT block. By considering this concept, instead of reducing the number of subcarriers directly, the incoming OFDM sequence $\mathrm{X}$ is partition into number of sub sequences. Set of each subsequence is considered as a group and the length of each group is less than the original number of sub carriers [11]. So $\widehat{N}$ is considered as the length of each group $(\widehat{N}<N)$.

Consider the incoming OFDM sequence ' $\mathrm{X}$ ' as,

$$
X=x_{0}, x_{1}, \ldots \ldots x_{\widehat{N}-1}, \ldots . x_{N-\widehat{N}}, \ldots x_{N-1}
$$

Then divide the elements in ' $\mathrm{X}$ ' and considered as different group. For easiness, here consider D number groups. Then $\widehat{N}=\frac{N}{D}$, and each group can be expressed as,

$$
X=x_{0}, x_{1}, \ldots \ldots x_{\widehat{N}-1} \text { and } X_{D}=x_{N-\widehat{N}}, \ldots \ldots \ldots x_{N-1}
$$

Fig 2 shows the transmitter section of new SLM method. Here, the number of elements in each group is less than that of ' $X$ '. So the number of sub carriers in each sub block is reduced and hence the complexity of IFFT block is reduced.
So computational complexity of the new system in terms of complex addition can be described as,

$$
\text { Comlexity }=U N \log _{2} \widehat{N}
$$

Where, $U$ is the number of phase sequence (newly generated sequence after the IFFT operation) and $\widehat{N}$ is the length of new group elements.

As per the conventional SLM, the subcarriers are given to the IFFT block only after the multiplication with the phase sequences. So here, each sub-sequence (each elements in different group) is want to be multiplied with the phase sequences. But if we use the phase sequence $b_{n}^{u}=\left(e^{-j \theta_{n}^{u}}\right)$ as same as the conventional SLM, information about the phase sequence want to be transmitted along with the original signal. So SI transmission is the next criteria in SLM technique. Hence the next modification is based on the phase sequence.

In the conventional SLM technique, the components of the phase sequences used to multiply have magnitude of 1 . So different data blocks varies only in phase. So here consider, some of the phase sequences component have magnitude larger than 1 while the other components of phase sequence have magnitude 1[12]. Hence, in the alternative OFDM data blocks a small number of data blocks are modified by the phase sequences.

In ordinary SLM the data block $X$ is multiplied carrier wise with $\mathrm{U}$ different phase sequences $B^{(U)}$ resulting the $\mathrm{U}$ different data blocks $X^{(U)}$, where $b_{n}^{u}=\left(e^{-j \theta_{n}^{u}}\right)$. But in my work instead of this phase sequence a modified phase sequence having magnitude greater than 1 is considered. So here consider the new phase sequence as, $b_{n}^{u}=\left(F e^{-j \theta_{n}^{u}}\right)^{k_{n}^{u}}$ where $k_{n}^{u}=0,1 ; \mathrm{n}=0,1 \ldots \mathrm{N}-1 ; \mathrm{u}=1,2 \ldots \mathrm{U}$ and $\mathrm{F}$ is the magnitude extension factor. After the multiplication the modified signal can be expressed as,

$$
X_{n}^{(u)}=X_{n}\left(F e^{-j \theta_{n}^{u}}\right)^{k_{n}^{u}}, k_{n}^{u}=1
$$

Then the magnitude of the modified signal is much greater than that of original. The value of magnitude extension factor must be real and greater than 1 . To recover the original signal at the receiver, $\mathrm{F}$ value must be carefully determined. 


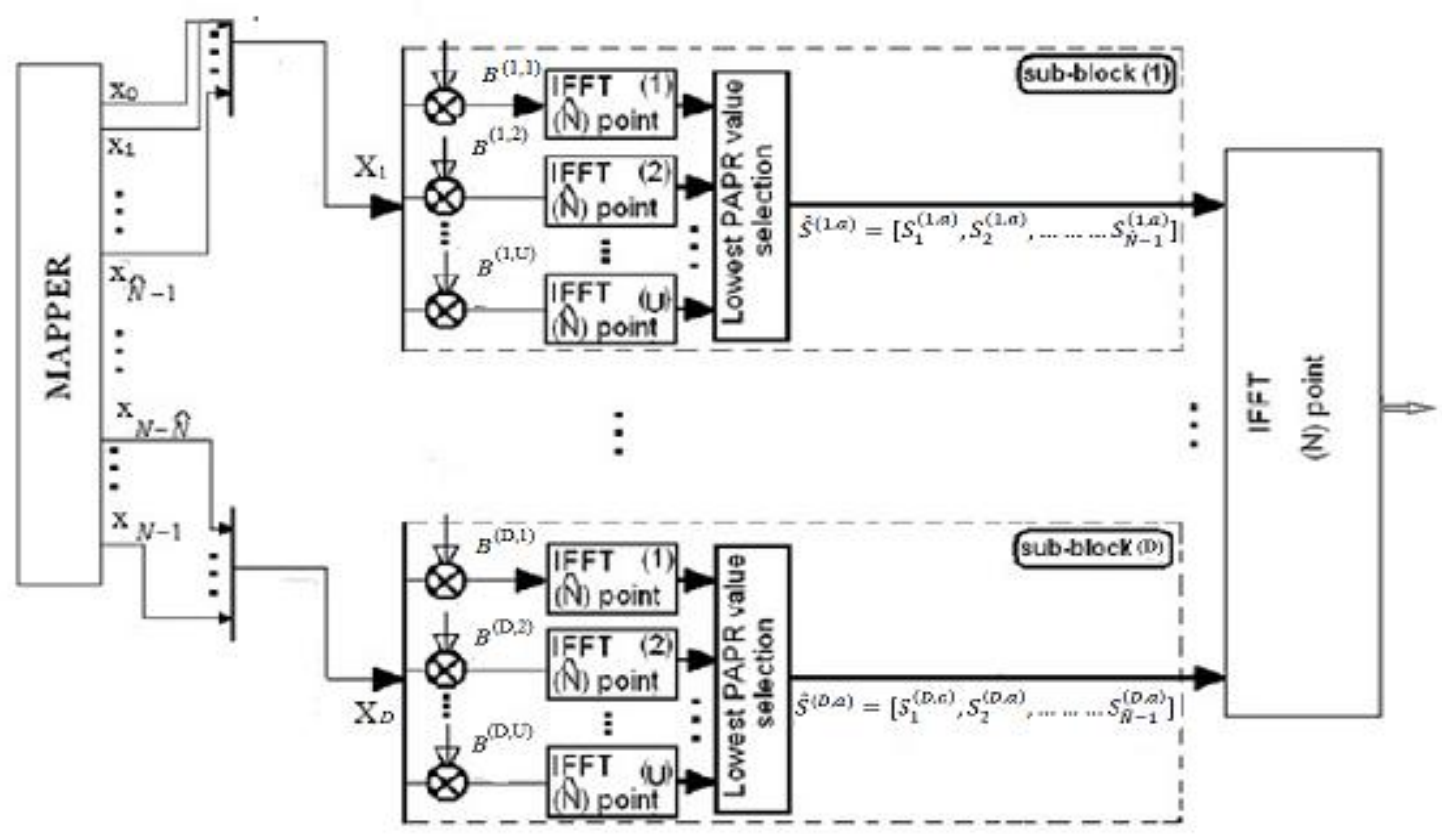

Fig 2: Transmitter of New SLM Technique

The convenient value for $\mathrm{F}$ should be determined so that the minimum distance between modified signal point and adjacent signal point in a constellation map is larger than the minimum distance between the original signal points in the constellation map. Otherwise the symbol error rate may increase. So, $d_{x}$ is considered as the minimum distance between the modified signal point and adjacent signal point in a constellation map and ' $2 d$ ' is considered as the minimum distance between the adjacent signal point in a constellation map.

For the proper recovery of the received signal, in an M- array QAM constellation $d_{x}$ should satisfy $d_{x} \geq 2 d$ with $F=$ $(((\sqrt{M}-1) \sqrt{2} d+d x)) /(\sqrt{2} d)$.

After the multiplication of sub sequence (subcarriers in each group) with these phase factor they are given to an IFFT block of length $\widehat{N}$ and the further processes are same as the conventional SLM. Consider, $U$ as the number of sequence generated from each sub sequence. From that the least PAPR value sequence is selected. This process is takes place in all individual sub block. Then the partially selected sequences are concatenated to form the final new sequence of length $\mathrm{N}$.
By considering this method, instead of using different IFFT blocks of length $\mathrm{N}$, we can use a IFFT block of length $\widehat{N}$ and hence the complexity is reduced. If the number of division as sub block is increased, there is a chance to degrade the performance of PAPR reduction, but the performance of that system gives a better performance than the ordinary SLM technique. Hence to get a good performance a moderate value of $\mathrm{D}$ can be selected.

Fig 3 shows the block diagram of receiver section of new SLM technique. In receiver section, we want to regenerate the phase sequence to identify whether a symbol in the subcarrier is modified or not. For that consider $\left(F e^{-j \theta_{n}^{u}}\right)^{k_{n}^{u}} \mathrm{n}$ with $k_{n}^{u}=0 \quad$ as unmodified and $k_{n}^{u}=1$ as modified component. So the phase sequence $\hat{b}_{n}=\left(F e^{-j \theta_{n}^{u}}\right)^{\hat{k}_{n}}$, are estimated as,

$$
\hat{k}_{n}= \begin{cases}0, & \text { for }\left|Y_{n}\right|<(F+\sqrt{M}-1) \frac{d}{\sqrt{2}} \\ 1, & \text { Otherwise }\end{cases}
$$

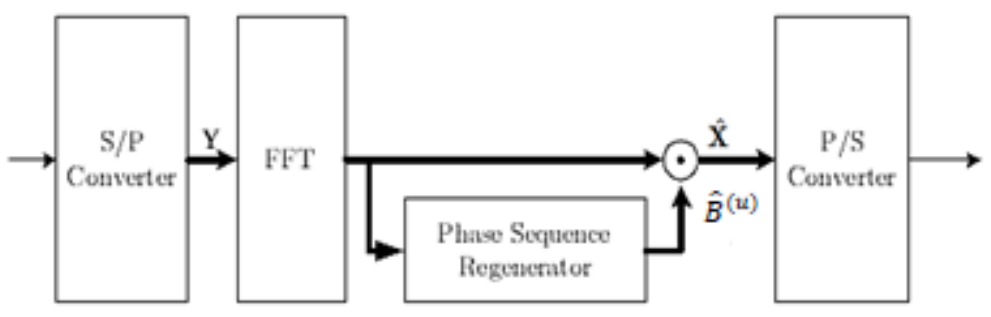

$\odot$ : Carriarwise divider

Fig 3: Receiver section of New SLM Technique 
After the regeneration of phase sequence $\hat{B}^{(u)}$, the received OFDM data block $Y=\left[Y_{0}, Y_{1} \ldots \ldots Y_{N-1}\right]$ is divided carrier wise by these phase sequence to obtain the estimate of the transmitted data block.

\section{SIMULATION RESULTS AND DISCUSSIONS}

In this work, computer simulation is implemented to evaluate the performance of the proposed method. The OFDM system was modeled using MATLAB. Complimentary cumulative distribution function (CCDF) of PAPR, power spectrum and BER versus SNR plots are used to measure the performance. Here, a simple AWGN channel is used as the channel model. An OFDM with 64 subcarriers with 16-QAM modulation is used. Also set the cyclic prefix as 4 .

For the complexity reduction, here the partitioning of 64 subcarriers as 4 groups is takes place. Also 4 phase sequence is used to multiply with the input sequence. So each group contains 16 subcarriers. Hence, for each group a 16 point IFFT is used. So as per the complexity equation, Complexity $=U N \log _{2} N$, complexity of the conventional SLM system is $1536[\mathrm{~N}=64$ and $\mathrm{U}=4]$, and this is reduced as 1024 in proposed method [ $\mathrm{N}=16$ and $\mathrm{U}=4]$.

Figure 4 shows the complexity values of SLM technique with 64 subcarriers partitioning in to different groups [2, 4, and 8].
Here 4 phase sequence are used to multiply with the input sequence

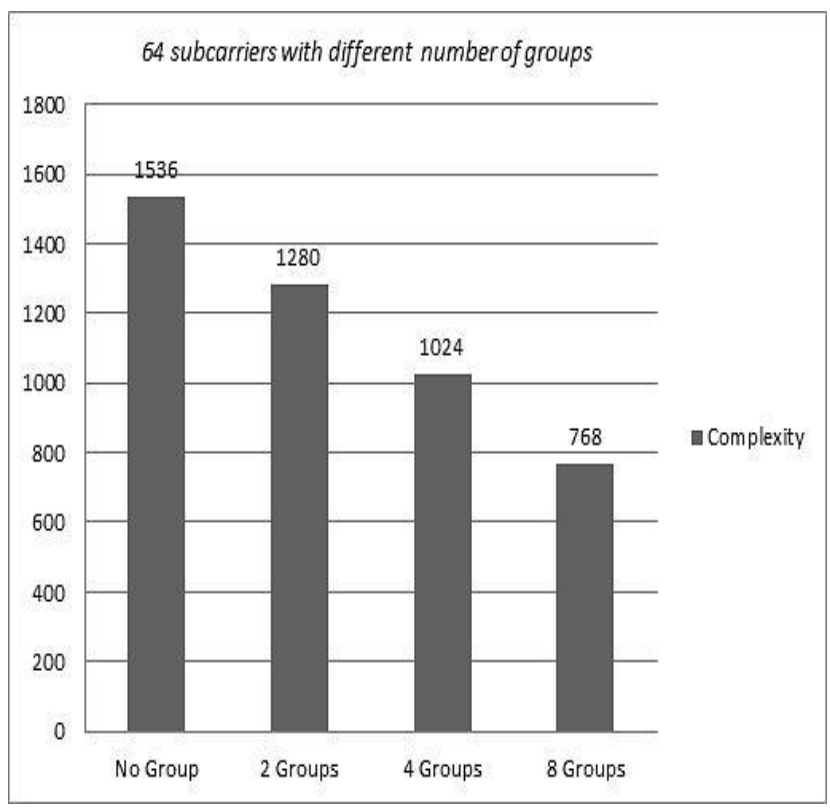

Fig 4: Complexity of SLM technique with different number of grouping

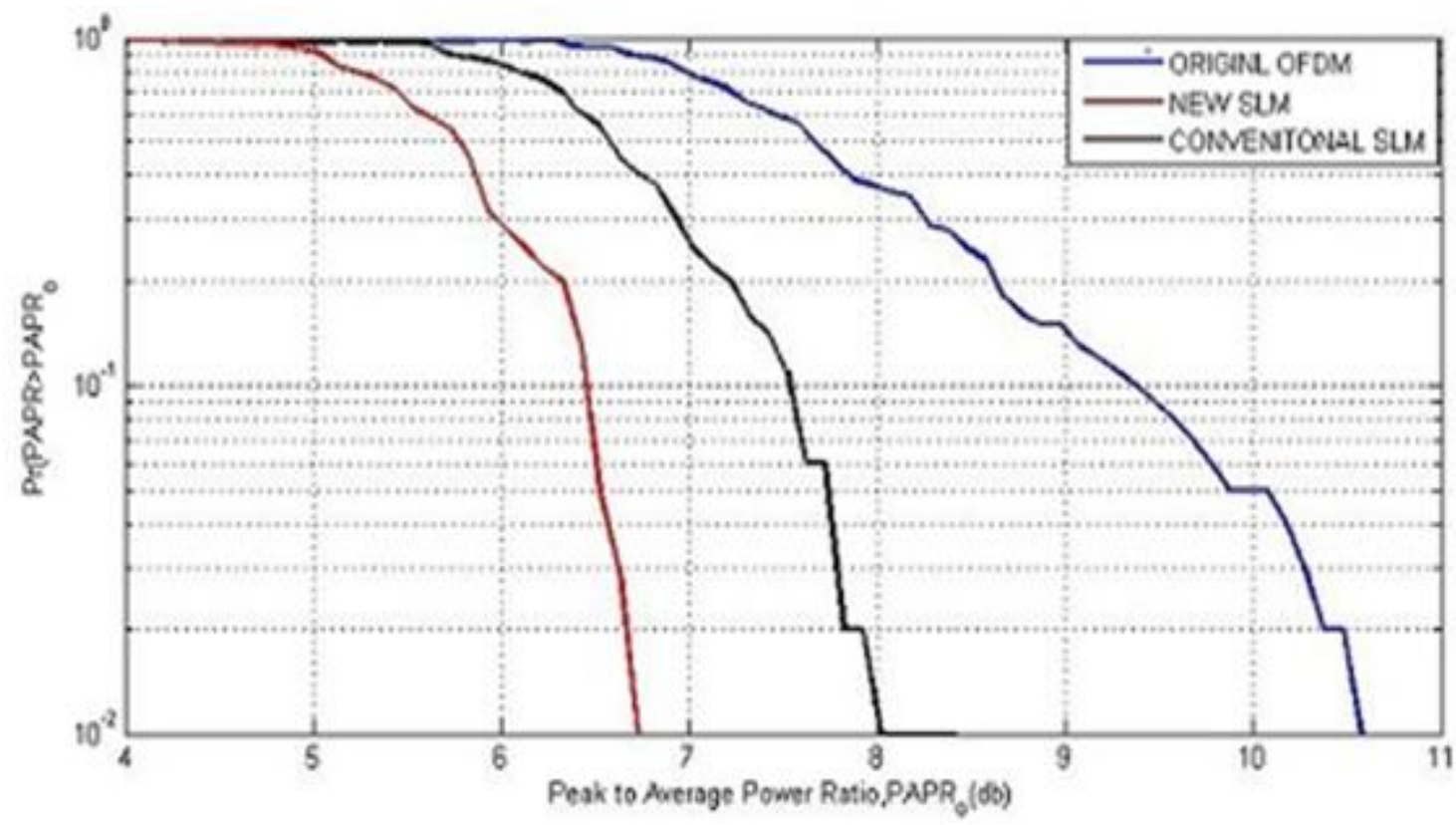

Fig 5: CCDF curve for new SLM, conventional SLM and original OFDM transmission with 64 sub carriers

Along with the complexity reduction here eliminate the transmission of side information by changing the phase value $\left(e^{-j \theta_{n}^{u}}\right)$ to $\left(F e^{-j \theta_{n}^{u}}\right)^{k_{n}^{u}}$, so the phase sequence has magnitude greater than 1.

Figure 5 represents the CCDF graph; it gives a comparison between original OFDM method, conventional SLM and the proposed SLM method. From the graph it is clear that to achieve the CCDF of .002 the proposed SLM method shows a $1.2 \mathrm{db}$ reduction in the threshold value than the conventional SLM.
Figure 6 gives the BER versus SNR graph it shows that, the proposed method have almost near BER value as the conventional method for SNR values up to 12.5. 


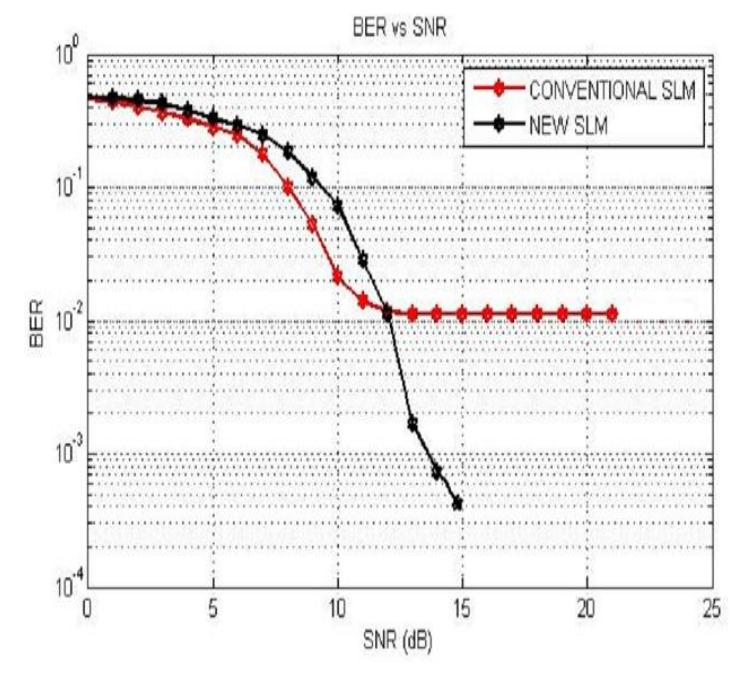

6: BER vs SNR

After that, to achieve higher SNR value new method gives better BER reduction

The PSD graph given in figure 7 shows that the proposed method has less power utilization than the original OFDM and same as the conventional SLM, hence the efficiency of power utilization is better.

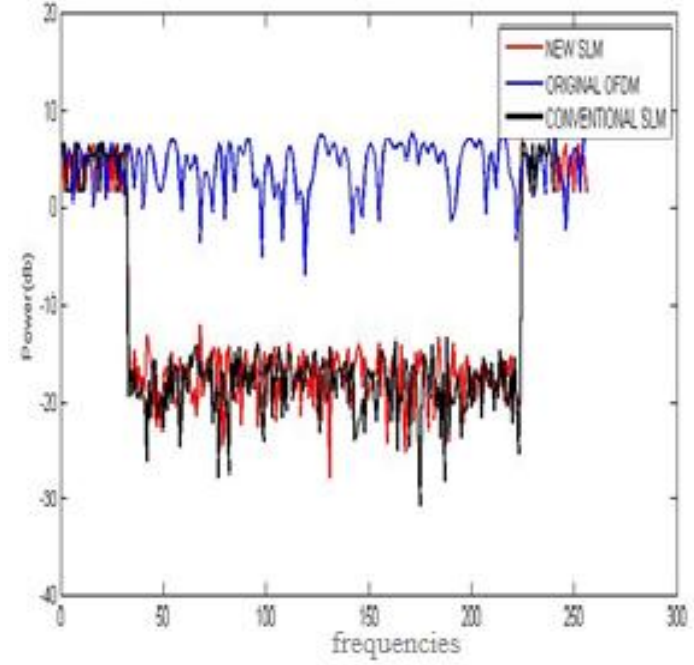

Fig 7: Power spectrum of the new SLM, conventional SLM and original OFDM

Below table shows the performance comparison between the original SLM method and the proposed method.

Table 1. Performance comparison of conventional and new SLM method

\begin{tabular}{|c|c|c|c|c|}
\hline Method & $\begin{array}{c}\text { PAPR(db) } \\
\text { (Comparison with } \\
\text { original OFDM 10.5 } \\
(\mathbf{d b}) \text { ) }\end{array}$ & $\begin{array}{c}\text { Complexity } \\
\boldsymbol{U N} \log _{2} N\end{array}$ & $\begin{array}{c}\text { SI } \\
\text { Transmission }\end{array}$ & $\begin{array}{c}\text { Power utilization } \\
\text { (comparison with } \\
\text { original } \\
\text { transmission) }\end{array}$ \\
\hline $\begin{array}{c}\text { Conventional } \\
\text { SLM }\end{array}$ & $2.5 \mathrm{db}$ reduction & {$[\mathrm{U}=4$ and N=64] } & Yes & Reduced \\
\hline New SLM & $3.9 \mathrm{db}$ reduction & $\begin{array}{c}1024 \\
{[\mathrm{U}=4 \text { and } \mathrm{N}=16]}\end{array}$ & No & Reduced \\
\hline
\end{tabular}

\section{CONCLUSION}

OFDM is a very attractive technique for multicarrier transmission and has become one of the standard choices for high speed data transmission over a communication channel due to its spectrum efficiency and channel robustness. One of the serious drawbacks of in OFDM systems is that the composite transmit signal can exhibit a very high PAPR when the input sequences are highly correlated. To avoid this PAPR, lots of works are already available in this field. SLM technique is one of the efficient techniques among them to reduce PAPR effectively. But this method has two drawbacks that are related to the computational complexity associated with the IFFT blocks and the transmission of side information.

So here a new method is proposed to overcome these drawbacks. This new method can reduce the computational complexity of the system by reducing the iteration of IFFT blocks through the partitioning of subcarriers as small groups. By using a phase sequence having magnitude greater than 1, this method eliminates the transmission of phase sequence as side information. This method also provides a better reduction in PAPR with better power efficiency.
The proposed SLM technique can also be verified in the multiuser OFDM system. For that, the input data sequence in each transmitter is divided into subgroups and multiplied with the modified phase sequence.

\section{REFERENCES}

[1] Seung hee han, Stanford university, Jae hong lee, Seoul national university. ,"An Overview of Peak-to-Average Power Ratio Reduction Techniques for Multicarrier Transmission, IEEE Wireless Communications, April 2005.

[2] Tao Jiang, Member, IEEE, and Yiyan Wu, Fellow, IEEE, An Overview: Peak to-Average Power Ratio Reduction Techniques for OFDM Signals, IEEE Transactions on Broadcasting, vol. 54, no. 2, June 2008.

[3] Andrea Gold Smith, a text book on "wireless communication".

[4] Dae-Woon Lim, Seok-Joong Heo, and Jong- Seon No, An Overview of Peak-to-Average Power Ratio reduction Schemes for OFDM Signals, Journal of Communications and Networks, vol. 11, no. 3, June 2009. 
[5] Sanjay Singh, M.Sathish Kumar and H.S.Mruthyunjaya, Effect of Peak-to-Average Power Ratio Reduction on the Multicarrier Communication System Performance Parameters, International Journal of Electrical and Computer Engineering, Dec 2009.

[6] Md. Ibrahim Abdullah, Md. Zulfiker Mahmud, Md. Shamim Hossain, Md. Nurul Islam, Comparative Study of PAPR Reduction Techniques in OFDM , ARPN Journal of Systems and Software, vol. 1, no. 8, Nov. 2011.

[7] Ashish K. Thakre, Snehal B. Meshram, Peak-to-Average Power Ratio Reduction in OFDM system using Block Coding technique, International Journal of Advanced Research in Computer and Communication Engineering ,Vol. 2, Issue

[8] R.W. Bauml, R.F.H. Fischer, and J.B. Huber. "Reducing the peak-to-average power ratio of multicarrier modulation by selected mapping", Electronics Letters, Oct. 1996.

[9] Ms. V. B. Malode, Dr. B. P. Patil ,'PAPR Reduction Using Modified Selective Mapping Technique", Int. J. of Advanced Networking and Applications, vol.02, Issue: 02, Aug 2010

[10] Leonard J. Cimini, Jr., Fellow, IEEE, and Nelson R. Sollen berger, Fellow, IEEE, Peak-to-Average Power Ratio Reduction of an OFDM Signal Using Partial Transmit Sequences, IEEE Communications Letters, vol. 4, no. 3, march 2000.
[11] Ibrahim Mohammad Hussain, Department of Computer Engineering, Sir Syed University of Engineering and Technology, "Low Complexity Partial SLM Technique for PAPR Reduction in OFDM Transmitters", International Journal on Electrical Engineering and Informatics, Vol. 5, no. 1, March 2013.

[12] Yang Chan Cho, Seung Hee Han, and Jae Hong Lee School of Electrical Engineering and Computer Science, Seoul National University, "Selected Mapping Technique with Novel Phase Sequences for PAPR Reduction of an OFDM Signal", IEEE Publication on Vehicular Technology Conference, Vol. 7, Sept. 2004.

[13] Gerhard Wunder, Robert F.H. Fisher, Holger Boche, Simon Litsyn and Jong- Seon No, "The PAPR Problem in OFDM Transmission”, IEEE Signal Processing Magazine, Nov 2013.

[14] Pawan Sharma, Department of Electronics and Communication, GGSIP University, Seema Verma," Performance Analysis of Peak-to-Average Power Ratio Reduction Techniques for Wireless Communication Using OFDM Signals ",IJCSI International Journal of Computer Science, Vol. 7, Issue 6, Nov. 2010.

[15] A. Ghassemi and T.A.Gulliver ,Department of Electrical and Computer Engineering, University of Victoria, Victoria, BC, Canada, Low-Complexity Distortion less Techniques for Peak Power Reduction in OFDM Communication Systems, Journal of Computer Networks and Communications, vol 2012, 